\title{
ON ISOMETRIES OF PRODUCT SETS
}

PAUL J. KELLY

1. Introduction. If $A$ is a metric set, and $A^{2}$ its cartesian product with itself, it is possible, in terms of the metric $A$, to metrize $A^{2}$ in many different ways. Thus, the problem of determining when $A^{2}$ isometric to $B^{2}$ implies $A$ isometric to $B$ can be attacked by placing limitations on the sets $A$ and $B$ or on the product metrics. The present paper proves that the isometry is implied when $A$ and $B$ are bounded and linear and the metric in $A^{2}$ and $B^{2}$ is determined by the use of a modified Minkowski gauge.

Also stated, without proof, are certain conditions under which the isometry of two product sets implies the isometry of the factors. The proofs for these results, given in the author's doctoral thesis, ${ }^{1}$ are long and differ only in detail from the one demonstrated.

\section{Definitions.}

(A) Metric set. $A$ is a metric set if to each pair of its element $a_{i}, a_{j}$ there corresponds a real, non-negative number $\rho\left(a_{i}, a_{j}\right)$ which is independent of the order of the elements, zero if and only if $a_{i}=a_{j}$, and which satisfies $\rho\left(a_{i}, a_{j}\right)+\rho\left(a_{j}, a_{k}\right) \geqq \rho\left(a_{i}, a_{k}\right)$.

(B) Isometry. Metric sets $A$ and $B$ are isometric (indicated by $A \equiv B)$ if there exists between them a 1-1 correspondence of elements, $T$, such that $\rho\left(a_{i}, a_{j}\right)=\rho\left(T\left(a_{i}\right), T\left(a_{j}\right)\right)$. The metric in $A$ and $B$ need not be the same, but in writing $\rho\left(a_{i}, a_{j}\right)$ we shall understand that $\rho$ is the metric for the set containing $a_{i}$ and $a_{j}$.

(C) Linear set. Linear, here, will mean a set isometric to a subset of the euclidean line.

(D) Bounded set. A metric set $A$ is bounded if $\rho\left(a_{i}, a_{j}\right)$ is equal to or less than some real number $R$ for all couples in $A$. If there exists a couple $a_{i}, a_{j}$, such that $\rho\left(a_{i}, a_{j}\right)=R$, the set assumes its bound.

(E) Modified Minkowski gauge. In the first quadrant of the cartesian plane let $C^{\prime}$ be a curve having nonzero intercepts. A curve $C$ consisting of $C^{\prime}$ together with the segments of the $x$ and $y$ axes which $C^{\prime}$ intercepts will be called a modified Minkowski gauge if the following properties hold: (1) $C$ is a simply connected, closed, convex curve; (2) if $P_{1}\left(x_{1}, y_{1}\right), P_{2}\left(x_{2}, y_{2}\right)$ are any two points in the first quadrant and the lines joining the origin to $P_{1}$ and $P_{2}$ cut $C$ in $P_{1}^{\prime}$ and $P_{2}^{\prime}$, respectively, then the relations $x_{1} \leqq x_{2}$, and $y_{1} \leqq y_{2}$ imply $O P_{1} / O P_{1}^{\prime}$

Received by the editors August 17, 1947.

${ }^{1}$ On isometric transformations, University of Wisconsin, 1942. 
$\leqq O P_{2} / O P_{2}^{\prime}$. It is easily shown, and we shall need to use, the fact that (1) and (2) imply that: (3) when $x_{1}<x_{2}$ and $y_{1}<y_{2}$ then $O P_{1} / O P_{1}^{\prime}$ $<O P_{2} / O P_{2}^{\prime}$. In addition to (1) and (2) the gauge will be called symmetric if : (4) $C$ is symmetric about the line $y=x$.

(F) Minkowski metrizations. Let $A$, with elements $a_{i}$, be any metric set. We use $C$ to metrize $A^{2}$ as follows. To a pair of elements $\left(a_{i}, a_{j}\right),\left(a_{k}, a_{l}\right)$ in $A^{2}$ there corresponds a metrizing point $P$ in the plane with coordinates $\left[\rho\left(a_{i}, a_{k}\right), \rho\left(a_{j}, a_{l}\right)\right]$. Let the line determined by $O$ and $P$ cut $C$ in the point $P^{\prime}$. Then $\rho\left[\left(a_{i}, a_{j}\right),\left(a_{k}, a_{l}\right)\right]$ is defined as the ratio $O P / O P^{\prime}$. We shall also use the notation $\|P\|$ for $O P / O P^{\prime}$. Thus condition (2) under (E) could be stated that when $x_{1} \leqq x_{2}$ and $y_{1} \leqq y_{2}$ then $\left\|\left(x_{1}, y_{1}\right)\right\| \leqq\left\|\left(x_{2}, y_{2}\right)\right\|$.

It can be shown that if $C$ has properties (1) and (2) under (E) then $A^{2}$ will satisfy definition $(A)$ of a metric set. ${ }^{2}$ Some condition similar to (2) is essential, for the metric defined this way with an ordinary Minkowski gauge permits examples in which $A^{2}$ is not a metric set. Symmetry about $y=x$ is not a necessary but a natural condition to assume in dealing with square sets. Since $\|P\|=1$ if and only if $P$ lies on $C, C$ is often referred to as a unit gauge. Various familiar metrics derive in a natural way from different choices of the gauge. If $C^{\prime}$ is the arc of a unit circle then $\|(x, y)\|=\left(x^{2}+y^{2}\right)^{1 / 2}$. If $C$ is a unit square, then $\|(x, y)\|=\operatorname{Max}(x, y)$. If $C^{\prime}$ is the line joining $(1,0)$ and $(0,1)$ then $\|(x, y)\|=x+y$.

Theorem 1. If $A$ and $B$ are bounded linear sets which assume their bounds and if $A^{2}, B^{2}$ are metrized by the same symmetric gauge $C$, then $A^{2} \equiv B^{2}$ implies $A \equiv B$.

Proof. (1) Let $T$ be the correspondence establishing $A^{2} \equiv B^{2}$. Let $M_{1}$ and $M_{2}$ be the bounds of $A$ and $B$ respectively. From linearity these bounds are assumed at one and only one pair of elements in each set. Let $\rho\left(\bar{a}, a^{*}\right)=M_{1}, \rho\left(\bar{b}, b^{*}\right)=M_{2}$.

(2) Let $P_{1}, P_{2}, P_{3}, P_{4}$ in $A^{2}$ be the points $(\bar{a}, \bar{a}),\left(a^{*}, \bar{a}\right)\left(a^{*}, a^{*}\right)$, $\left(\bar{a}, a^{*}\right)$ and $Q_{1}, Q_{2}, Q_{3}, Q_{4}$ in $B^{2}$ have similar coordinates with " $a$ " replaced by "b." Let $T\left(P_{i}\right)=S_{i}=\left(u_{i}, v_{i}\right), i=1,2,3,4$.

(3) The set $\left\{S_{i}\right\}$ is in some order the set $\left\{Q_{i}\right\}, i=1,2,3,4$. We prove this by showing each $u_{i}$ and each $v_{i}$ is $b$ or $b^{*}$ as follows.

(3.1) From the coordinates of $P_{i}$ and the definition of the metric in $A^{2}$ we have $\rho\left(P_{1}, P_{3}\right)=\rho\left(P_{2}, P_{4}\right)=\left\|\left(M_{1}, M_{1}\right)\right\|$ and $\rho\left(Q_{1}, Q_{3}\right)$ $=\rho\left(Q_{2}, Q_{4}\right)=\left\|\left(M_{2}, M_{2}\right)\right\|$. From property (2) under (E) these must be the largest distances which occur in $A^{2}$ and $B^{2}$ respectively. Then

2 Ibid. 
from $A^{2} \equiv B^{2}$, we have $\left\|\left(M_{1}, M_{1}\right)\right\|=\left\|\left(M_{2}, M_{2}\right)\right\|$. Since $\left(M_{1}, M_{1}\right)$ and $\left(M_{2}, M_{2}\right)$ both lie on $y=x$ and this line cuts $C^{\prime}$ in only one point it follows that $M_{1}=M_{2}$. Let $M_{1}=M_{2}=M$. From symmetry of $C$ the $x$ and $y$ intercepts of $C^{\prime}$ are equal and for simplicity we may take them as $(M, 0)$ and $(0, M)$. (This could always be done by a radial expansion or contraction of $C$ that would change all distances in $A^{2}$ and $B^{2}$ by the same multiple.) With this condition it follows from (1) and (2) that $\rho\left(P_{1}, P_{2}\right)=\rho\left(P_{2}, P_{3}\right)=\rho\left(P_{3}, P_{4}\right)=\rho\left(P_{4}, P_{1}\right)=1$.

From (3.1) and $A^{2} \equiv B^{2}$ we have

$$
\rho\left(S_{1}, S_{2}\right)=\rho\left(S_{2}, S_{3}\right)=\rho\left(S_{3}, S_{4}\right)=\rho\left(S_{4}, S_{1}\right)=1,
$$

and

$$
\rho\left(S_{1}, S_{3}\right)=\rho\left(S_{2}, S_{4}\right)=\|(M, M)\| .
$$

Now $\|(M, M)\| \geqq 1$ and we treat these two possibilities separately. (3.4) Case 1. $\|(M, M)\|>1$. Then if $\bar{P}(\bar{x}, \bar{x})$ is the gauge point on $y=x$ (that is, intersection of $C^{\prime}$ and $y=x$ ), we have $0<\bar{x}<M$. From (1) we have

$$
\rho\left(u_{1}, u_{3}\right) \leqq M, \quad \rho\left(v_{1}, v_{3}\right) \leqq M
$$

and

$$
\rho\left(u_{2}, u_{4}\right) \leqq M, \quad \rho\left(v_{2}, v_{4}\right) \leqq M .
$$

From (3.3) and property (3) of the gauge $C$, one of the equalities in (3.5) and one in (3.6) must hold. To show that all four equalities must hold, suppose:

(3.7) $\rho\left(u_{1}, u_{3}\right)=M, \rho\left(v_{1}, v_{3}\right)<M$. Let $R$ be the point $\left[\rho\left(u_{1}, u_{3}\right)\right.$, $\left.\rho\left(v_{1}, v_{3}\right)\right]$. From $\left\|\rho\left(u_{1}, u_{3}\right), \rho\left(v_{1}, v_{3}\right)\right\|=\|(M, M)\|$ it follows, using similar triangles, that the gauge point $R^{\prime}$ on the line joining $O$ and $R$ lies on the line $x=\bar{x}$. Since the points $\bar{P}$ and $(M, O)$ are on $C$, the chord joining them must lie on or interior to $C$. Hence, $R^{\prime \prime}$, the intersection of this chord with the radial line through $R$, must lie on or interior to $C$, which is impossible since $O R^{\prime \prime}>O R^{\prime}$. This contradiction shows that if $\rho\left(u_{1}, u_{3}\right)=M$ then $\rho\left(v_{1}, v_{3}\right)=M$. The four possibilities of one inequality holding in (3.5) and (3.6) lead to a similar contradiction, hence all four equalities hold. But from (1) that implies each $u_{i}$ and each $v_{i}, i=1,2,3,4$, is either $\bar{b}$ or $b^{*}$ and establishes (3).

(3.8) Case 2. $\|(M, M)\|=1$. Then $(M, M)$ as well as $(M, O)$ and $(O, M)$ are gauge points. From the definition of convexity then there can be no gauge point interior to the square $(O, O),(M, O),(M, M)$, $(O, M)$. But now from (3.2) and (3.3) all six distances in the set $\left\{T\left(P_{i}\right)\right\}, i=1,2,3,4$, are 1 , therefore the metrizing point correspond- 
ing to any pair of these elements must lie on $C$, and hence must have either its 1 st or 2 nd coordinate equal to $M$. Then either $\rho\left(u_{i}, u_{j}\right)=M$ or $\rho\left(v_{i}, v_{j}\right)=M, i, j=1,2,3,4, i \neq j$. Now assume $v_{1} \neq b$ or $b^{*}$. Then $\rho\left(v_{1}, v_{2}\right) \neq M, \rho\left(v_{1}, v_{3}\right) \neq M, \rho\left(v_{1}, v_{4}\right) \neq M$. Therefore, $\rho\left(u_{1}, u_{2}\right)=\rho\left(u_{1}, u_{3}\right)$ $=\rho\left(u_{1}, u_{4}\right)=M$. Take $u_{1}=\bar{b}$, then $u_{2}=u_{3}=u_{4}=b^{*}$. Therefore $\rho\left(u_{2}, u_{3}\right)$ $=\rho\left(u_{2}, u_{4}\right)=\rho\left(u_{3}, u_{4}\right)=0$. Then $\rho\left(v_{2}, v_{4}\right)=\rho\left(v_{2}, v_{3}\right)=\rho\left(v_{3}, v_{4}\right)=M$. Let $v_{2}=b$ then $v_{3}=v_{4}=b^{*}$, and $\rho\left(v_{3}, v_{4}\right)=0$ contradicting $\rho\left(v_{3}, v_{4}\right)=M$. Therefore $v_{1}$ must equal $\bar{b}$ or $b^{*}$ and by the same argument each $u_{i}$ and each $v_{i}, i=1,2,3,4$, must be $\bar{b}$ ar $b^{*}$, which establishes (3).

(4) If we think of $P_{1}, P_{3}$ and $P_{2}, P_{4}$ as diagonal pairs in $A^{2}$ and $Q_{1}, Q_{3}$ and $Q_{2}, Q_{4}$ as diagonal pairs in $B^{2}$ it is clear, since there are six pairs in each set, that $T$ must map at least one nondiagonal pair of the $P_{i}$ 's onto a nondiagonal pair of the $Q_{i}$ 's. The symmetry of $C$ makes the argument the same in any case and we may suppose for definiteness that $T\left(P_{1}\right)=Q_{1}, T\left(P_{2}\right)=Q_{2}$.

We define a mapping, $S$, of $A$ onto $B$ as follows. To any element $a_{i}$ in $A$ there corresponds in $A^{2}$ the point $P_{i}\left(a_{i}, \bar{a}\right)$. Let $T\left(P_{i}\right)=Q_{i}$ $=\left(b_{i}, b_{k}\right)$, that is, label the first coordinate of the transformed point $b_{i}$. Define $S$ by $S\left(a_{i}\right)=b_{i}, S(\bar{a})=\bar{b}, S\left(a^{*}\right)=b^{*}$.

(5) $S$ establishes $A \equiv B$.

(5.1) From the coordinates of $P_{1}, P_{i}, P_{2}$ we have $\rho\left(P_{1}, P_{i}\right)$ $=\rho\left(\bar{a}, a_{i}\right) / M, \rho\left(P_{i}, P_{2}\right)=\rho\left(a_{i}, a^{*}\right) / M, \rho\left(P_{1}, P_{2}\right)=\rho\left(\bar{a}, a^{*}\right) / M$.

(5.2) Linearity in $A$ gives $\rho\left(\bar{a}, a_{i}\right)+\rho\left(a_{i}, a^{*}\right)=\rho\left(\bar{a}, a^{*}\right)$.

(5.3) (5.1) and (5.2) imply $\rho\left(P_{1}, P_{i}\right)+\rho\left(P_{i}, P_{2}\right)=\rho\left(P_{1}, P_{2}\right)$.

(5.4) From (5.3) and $A^{2} \equiv B^{2}$ we have $\rho\left(Q_{1}, Q_{i}\right)+\rho\left(Q_{i}, Q_{2}\right)$ $=\rho\left(Q_{1}, Q_{2}\right)$.

(5.5) Consider the point $\bar{Q}_{i}\left(b_{i}, \quad b\right)$. From the coordinates of $Q_{1}, \bar{Q}_{i}, Q_{2}$ and linearity in $B$ it follows that $\rho\left(Q_{1}, \bar{Q}_{i}\right)+\rho\left(\bar{Q}_{i}, Q_{2}\right)$ $=\rho\left(Q_{1}, Q_{2}\right)$.

(5.6) (5.4) and (5.5) give $\rho\left(Q_{1}, Q_{i}\right)+\rho\left(Q_{i}, Q_{2}\right)=\rho\left(Q_{1}, \bar{Q}_{i}\right)+\rho\left(\bar{Q}_{i}, Q_{2}\right)$.

(5.7) The metrizing point corresponding to the couple $Q_{1}, Q_{i}$ has its coordinates respectively equal to or greater than those for the point corresponding to the couple $Q_{1}, \bar{Q}_{i}$. Hence, $\rho\left(Q_{1}, Q_{i}\right) \geqq \rho\left(Q_{1}, \bar{Q}_{i}\right)$. Similarly $\rho\left(Q_{i}, Q_{2}\right) \geqq \rho\left(\bar{Q}_{i}, Q_{2}\right)$. These relations together with (5.6) imply $\rho\left(Q_{1}, Q_{i}\right)=\rho\left(Q_{1}, \bar{Q}_{i}\right)$ and $\rho\left(Q_{i}, Q_{2}\right)=\rho\left(\bar{Q}_{i}, Q_{2}\right)$.

(5.8) Since $\rho\left(Q_{1}, \bar{Q}_{i}\right)=\rho\left(b, b_{i}\right) / M$, from (5.1), $A^{2} \equiv B^{2}$ and (5.7) we obtain $\rho\left(\bar{a}, a_{i}\right)=M \rho\left(P_{1}, P_{i}\right)=M \rho\left(Q_{1}, Q_{i}\right)=M \rho\left(Q_{1}, \bar{Q}_{i}\right)=\rho\left(\bar{b}, b_{i}\right)$.

(5.9) Let $a_{i}, a_{j}$ be any two elements in $A$ with $b_{i}, b_{j}$ their transforms under $S$. From (5.8) we have $\rho\left(\bar{a}, a_{j}\right)=\rho\left(\bar{b}, b_{j}\right)$ and $\rho\left(\bar{a}, a_{i}\right)$ $=\rho\left(b, b_{i}\right)$, and from the linearity of $A$ and $B$ it follows that $\rho\left(a_{i}, a_{j}\right)$ $=\rho\left(b_{i}, b_{j}\right)$. This last implies that if $a_{i} \neq a_{j}$ then $b_{i} \neq b_{j}$ so the mapping cannot be many-to-one. Also $S$ is not a mapping of $A$ onto a part of $B$ 
because, using the same method, we could define an isometric transform $S^{\prime}$ of $B$ onto $A$, then $S S^{\prime}$ would be an isometry of $A$ onto a subset of itself which is impossible. Thus $S$ is $1-1$ and (5) is established.

3. Other results. If $A_{1}, A_{2}, \cdots, A_{n}$ are bounded linear sets which assume their bounds, they do so only on one pair of elements which might be called end points. In the product set $A_{1} \times A_{2} \times \cdots \times A_{n}$ there are $2^{n} n$-tuples each coordinate of which is an end point of a base set. Call this collection of points in the product the vertex set. In the following theorems, as in the proof just given, the method employed was to show that the mapping giving the isometry of two product sets had to map the vertex set of one into the vertex set of the other. It was then possible, as in the proof of Theorem 1, to use the isometry of the products to establish the isometry of the base sets.

If $P_{1}\left(a_{11}, a_{12}, \cdots, a_{1 n}\right), P_{2}\left(a_{21}, a_{22}, \cdots, a_{2 n}\right)$ are two elements in $A_{1} \times A_{2} \times \cdots \times A_{n}$, then by the euclidean, maximum, and sum metrics in the product we shall mean that $\rho\left(P_{1}, P_{2}\right)$ is given respectively by $\left[\sum \rho\left(a_{1 i}, a_{2 i}\right)^{2}\right]^{1 / 2}, \operatorname{Max}\left[\rho\left(a_{11}, a_{21}\right), \rho\left(a_{12}, a_{22}\right), \cdots\right.$. $\left.\rho\left(a_{1 n}, a_{2 n}\right)\right]$, or by $\sum \rho\left(a_{1 i}, a_{2 i}\right)$.

Theorem 2. If $A_{1}, A_{2}, B_{1}, B_{2}$ are bounded, linear sets assuming nonzero bounds and if $A_{1} \times A_{2}$ and $B_{1} \times B_{2}$ are both metrized under (1) the sum metric or (2) the maximum metric, then $A_{1} \times A_{2} \equiv B_{1} \times B_{2}$ implies $A_{1} \equiv B_{1}, A_{2} \equiv B_{2}$ or $A_{1} \equiv B_{2}, A_{2} \equiv B_{1}$.

THEOREM 3. If $A$ is a bounded linear set, assuming its bound, and $B$ is a metric set, then if $A^{n}$ and $B^{n}$ are metrized by the maximum metric $A^{n} \equiv B^{n}$ implies $A \equiv B$.

Theorem 4. If $A_{1}, A_{2}, \cdots, A_{n}$ are linear, metric sets, each assuming a nonzero bound and if $B_{1}, B_{2}, \cdots, B_{n}$ are metric sets bounded from zero (not consisting of a single point) and if $A=A_{1} \times A_{2} \times \cdots \times A_{n}$ and $B=B_{1} \times B_{2} \times \cdots \times B_{n}$ are metrized by the euclidean metric then $A \equiv B$ implies that the sets $A_{i}$ are isometric to the sets $B_{i}$ in some order.

Theorem 5. Let $A_{1}, A_{2}, B_{1}, B_{2}$ be euclidean plane sets, each of which contains the vertices of some rectangle bounding it, and let $A_{1} \times A_{2}$ and $B_{1} \times B_{2}$ be metrized by the euclidean metric. If one of the sets $A_{1}, A_{2}$ is not isometric to a product of two linear sets, then $A_{1} \times A_{2} \equiv B_{1} \times B_{2}$ implies the sets $A_{i}$ are isometric in some order to the sets $B_{i}, i=1,2$.

University of Southern California 